\title{
Smiles 3D: Plataforma Web para Comunicação de Profissionais na Área Odontológica
}

\author{
Breno A. Fróes Peres', Guilherme Aguiar², \\ Claudio Fernandes ${ }^{2}$, Flávio Seixas ${ }^{1}$, Debora C. Muchaluat-Saade ${ }^{1}$ \\ ${ }^{1}$ Laboratório MídiaCom - Instituto de Computação \\ ${ }^{2}$ Faculdade deOdontologia- Campus de Nova Friburgo \\ Universidade Federal Fluminense - Niterói, RJ \\ \{breno_froes, guilherme_aguiar, claudiofernandes\}@id.uff.br, \\ $\{$ fseixas, debora\} @midiacom.uff.br
}

\begin{abstract}
With the new digital age, new needs are emerging in the dental market related to infrastructure, specializations or negotiation models. In order to meet such needs, Smiles 3D Project was created aiming at facilitating communication between prosthetists and dentists, improving offer and acquisition of dental services and products. In addition, it enables a more practical and quick commercial, educational or economic relationship for odontology professionals, making the market more competitive.

Resumo. Com a nova era digital, estão surgindo novas necessidades no mercado odontológico, sejam elas relacionadas a infraestrutura, especializações ou modelos de negociação. Dessa forma, para atender a tais necessidades, o projeto Smiles $3 D$ foi criado, com o intuito de facilitar a comunicação entre protéticos e dentistas, visando melhorar a oferta e a aquisição de serviços e produtos odontológicos. Além disso, possibilita um relacionamento mais prático e rápido, seja comercial, educacional ou econômico, na área odontológica, tornando o mercado mais competitivo.
\end{abstract}

\section{Introdução}

Dentistas têm passado por um problema recorrente para encontrar protéticos que possam atender a suas demandas de serviços, com uma quantidade limitada de contatos. Além disso, as distâncias entre os profissionais muitas vezes dificultam vertiginosamente o fechamento de negócios e atrasam o prazo dado pelo dentista em seus serviços, afetando o paciente final.

Dessa maneira, nasce uma carência na área odontológica, no que diz respeito a dentistas encontrarem profissionais protéticos capazes de realizar as exigências dos seus serviços e também dos protéticos encontrarem serviços em que são habilitados e que estão dispostos a realizar.

Para atender a tais exigências do mercado, foram criadas plataformas capazes de estabelecer as devidas comunicações e então saciar o desprovimento de aplicações capazes de interligar esses profissionais, como a Booklab, aplicativo que estabelece relações comerciais práticas entre dentistas, protéticos autônomos e laboratórios de próteses [Plataforma Booklab, 2019]. Entretanto, tais plataformas são de difícil acesso para iniciantes em suas respectivas carreiras odontológicas, para novos laboratórios ou mesmo para dentistas e protéticos experientes, mas que não possuem capital necessário para investir em tais aplicações por exigirem custos altos. 
Atualmente, para que protéticos possam ser encontrados por dentistas, eles precisam estar registrados em uma rede de conexão que, por muitas vezes, é excessivamente arcaica, ineficaz e onerosa. $\mathrm{Na}$ prática, existe uma dificuldade de comunicação, com inúmeros ruídos e faltas de compreensões plenas de ambas as partes, ademais é restrita a grandes empresas, profissionais e corporações.

Essas inconveniências acarretam um mercado pouco acessível e seletivo, atrasando a competitividade em toda a área odontológica, incapacitando o crescimento de dentistas iniciantes e de laboratórios de pequeno porte e, consequentemente, afetando o paciente final. Essa realidade encarece serviços e eleva prazos, os estagnando em seus âmbitos competitivos e os tornando ainda mais inacessíveis na área da saúde dental.

Este trabalho apresenta a ferramenta Smiles 3D, uma plataforma web para facilitar a comunicação entre dentistas e protéticos, visando ampliar a oferta e aquisição de serviços e produtos odontológicos.

O restante do texto está organizado da seguinte forma. Na Seção 2, plataformas web na área da saúde são comentadas. Na Seção 3, a plataforma Smiles 3D é apresentada de forma detalhada, salientando o objetivo do projeto e suas funcionalidades principais. Já na Seção 4, a interface atual do sistema é apresentada e suas respectivas funções retratadas. Na Seção 5, são expostos resultados sobre o teste de usabilidade realizado por profissionais da área odontológica. Finalmente, na Seção 6, conclui-se o trabalho e são vislumbradas as metas futuras. Ademais, na Seção 7 está a bibliografia deste texto e na Seção 8, o vídeo de utilização e tutorial da plataforma.

\section{Plataformas web na área de saúde}

Odonto Company [Plataforma Odonto Company, 2019] é uma plataforma com design impecável e ótima fluidez de funcionalidades, que consiste na busca de profissionais na área odontológica em que o usuário fornece seus dados para que haja o agendamento de consultas com parceiros da marca. O objetivo da rede de clínicas é deixar mais acessível a todos os cidadãos o tratamento odontológico, e possibilitar encontrar na plataforma especialistas capazes de atender a suas necessidades com a melhor qualidade e melhor custo.

Dr. Sintomas [Plataforma drsintomas, 2019] é uma aplicação web que realiza busca de sintomas em banco de dados alimentado por médicos, a fim de que forneça ao usúario a informação da doença ou do mal-estar em questão. O intuito é oferecer a quem acessa uma noção sobre o que tem e assim indicar um direcionamento sobre qual médico especialista buscar.

Zenklub [Plataforma Zenklub, 2019] é uma plataforma utilizada para facilitar o acesso aos melhores profissionais na área de saúde, minimizando complicações e dificuldades quando o assunto é encontrar um psicólogo ideal e mais próximo do usuário. A plataforma visa proporcionar aos pacientes serviços práticos, seguros e alta qualidade.

\section{Smiles 3D}

A plataforma Smiles 3D, proposta neste artigo, visa suprir a necessidade de uma comunicação eficaz entre profissionais na área da saúde odontológica. 
Como é definido em [Fernandes, 2005], “pregão é o procedimento administrativo por meio do qual a Administração Pública, garantindo a isonomia, seleciona fornecedor ou prestador de serviço, visando a execução de objeto comum no mercado, permitindo aos licitantes, em sessão pública, reduzir o valor da proposta por meio de lances verbais e sucessivos.” A plataforma Smiles 3D utiliza o método de pregões na modalidade privada.

O projeto de Smiles 3D tem como objetivo criar uma aplicação web, que deve juntar contatos de protéticos e de projetistas para buscas futuras para os dentistas e, além disso, oferecer a devida oferta de serviços odontológicos de forma direta e simples. Em relação aos protéticos e aos projetistas, a aplicação deve facilitar encontrar clientes dentistas que buscam serviços que os mesmos podem oferecer.

Esse projeto se propõe a desenvolver uma plataforma de serviços web para promover o uso de tecnologias digitais na odontologia, disponibilizados sob a forma de um portal especializado. O público alvo envolve dentistas, professores, protéticos, clinicas, lojas de varejo, fábricas de insumos e equipamentos, além de financeiras e empresas de logística de entrega, em um momento posterior. A concepção completa do produto compreende dois grupos de serviços principais a serem oferecidos, a parte acadêmica, voltada para treinamento, educação e discussão dos participantes, e a parte e-marketing, direcionado a assessoria, mercado digital, divulgação e serviços online dos usuários.

Logo, o objetivo essencial da Smiles 3D resume-se em estabelecer relações comerciais, sociais e profissionais, entre dentistas, protéticos e projetistas, compreendidos como usuários, de forma clara e substancialmente transparente, para ambas as partes e para os usuários externos e visitantes, porém, obviamente, com as devidas limitações de privacidade e segurança.

A aplicação web Smiles 3D realiza a troca de interesses quanto às próteses entre protéticos e dentistas através de pregões, realizados por dentistas e por quem busca por empresas ou profissionais independentes que possam ser capazes de oferecer a oferta com melhor custo benefício possível.

Na criação do pregão em questão, alguns campos devem ser informados para que haja o entendimento e detalhamento corretos quando os usuários realizarem interações diversas. Para o intermédio dos fechamentos de negócios, são realizados nos pregões comentários que possuem como objetivos o esclarecimento de dúvidas e a estabilização de valores. Na versão atual, a etapa final consiste no dentista visualizar a oferta do protético, acessar o seu perfil e então entrar em contato para o fechamento do negócio em questão.

Todavia, quanto às previsões futuras do projeto, o usuário padrão fechará os devidos pregões na própria plataforma e então realizará o negócio pessoalmente, contudo com as devidas seguranças monetárias e sociais, na medida do possível, liberando pagamentos somente após o fim da negociação e entrega do que foi combinado e ofertado. Cada transação realizada através da plataforma será avaliada com notas de 1 a 5, de ambas as partes, para computar a reputação dos usuários. 


\section{Implementação Atual}

A plataforma Smiles 3D é baseada em web e faz uso de algumas ferramentas e linguagens para seu desenvolvimento. Dentre elas estão o Django, em sua versão 2.0, Python, em sua versão 3.6.0, SQLite, em sua versão 3, HTML, em sua versão 5, CSS 3, em sua versão e Bootstrap, em sua versão 4.

O framework central responsável pelo recebimentos e respostas das solicitações dos usuários, Django [Django, 2019], funciona de forma que, ao aceitar solicitações de URLs, há um retorno a elas de todo o HTML necessário para um navegador web renderizar a página em questão. Tal framework, desenvolvido em Python, segue o conhecido padrão MVC de desenvolvimento, model, camada em que o banco de dados é implementado, no caso SQLite em fase de produção local, view, onde está a interface do sistema em si e controller, camada que implementa as regras de negócio, onde os dados são processados da camada model e então repassados para a camada view.

A interface do Smiles 3D foi planejada para que o projeto seja guiado da melhor forma, a permitir a implementação gradual de suas funcionalidades, focando sempre na responsividade da plataforma.

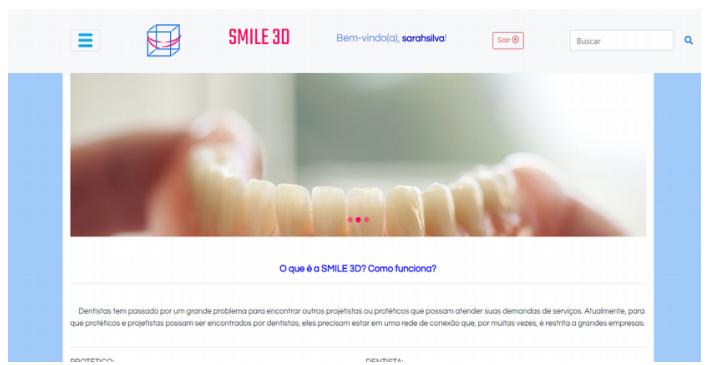

Figura 1. Tela inicial do site.

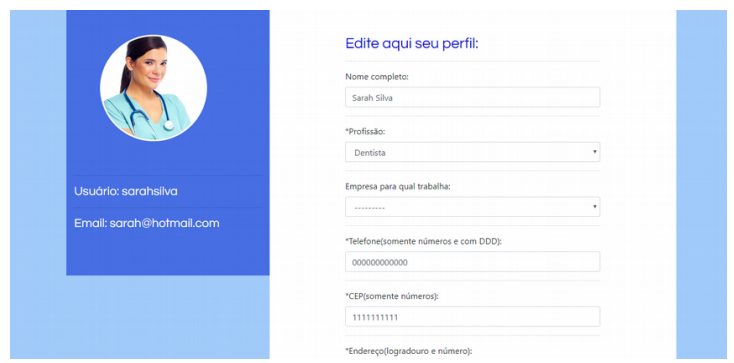

Figura 2. Tela para visualização de um perfil cadastrado como físico.

Na Figura 1, é mostrada a tela inicial da aplicação, em que o usuário pode saber mais sobre o Smiles 3D e conhecer do que se trata e em que situações a ferramenta pode ser utilizada. Através da tela inicial, o usuário faz seu cadastro na ferramenta, na versão atual, dentistas e protéticos estão disponíveis.

Na Figura 2,o usuário pode visualizar o seu perfil cadastrado e então editá-lo de acordo com suas necessidades, a permitir, por exemplo, colocar foto de perfil e também alterar a senha.

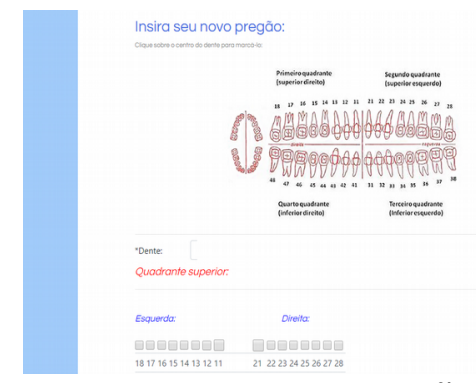

Figura 3. Tela em que são registrados os pregões.

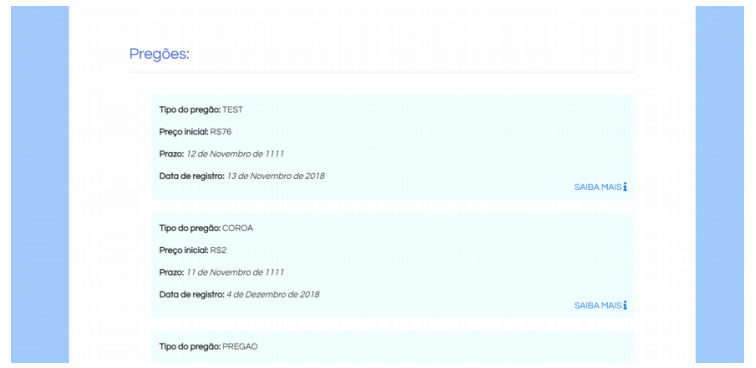

Figura 4. Tela em que são mostrados os pregões.

Na Figura 3, é exibida a tela que expõe a principal funcionalidade da aplicação, que no caso se trata da criação de pregões na plataforma, com os campos exigidos ou opcionais, como seleção dos dentes, prazo e orçamento. Tais pregões após seus 
registros, serão disponibilizados para demais usuários e então as ofertas serão efetuadas sobre ele.

Na Figura 4, são expostos todos os pregões registrados por todos os usuários, modalidade de licitação essa que efetivada, estará disponível para visualização tanto por usuários cadastrados na plataforma como por anônimos.

Já na Figura 5, é retratada a modelagem relacional do banco de dados SQLite da aplicação, feita através do software MySQL Workbench.

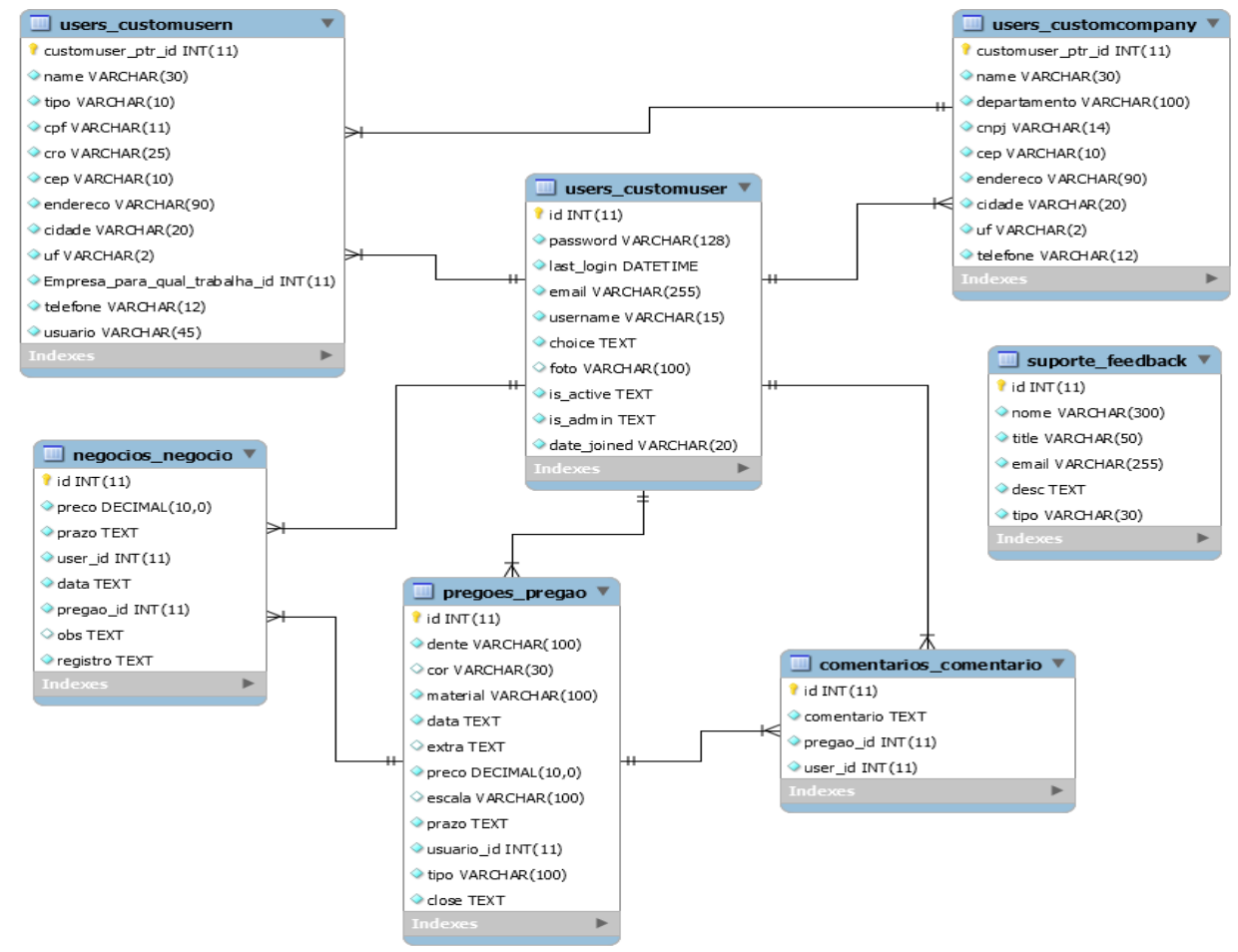

Figura 5. Tela do modelo relacional.

\section{Avaliação da Plataforma Smiles 3D}

Com o objetivo de avaliar a implementação atual da plataforma Smiles 3D, foi utilizado o padrão System Usability Scale (SUS) (Brooke, 1996). O SUS indica um questionário com dez perguntas sobre usabilidade de um sistema, que são respondidas usando a escala Likert, dando uma visão geral da facilidade de uso (ou falta dela) de sites, software, hardware, dispositivos móveis e outras aplicações tecnológicas.

O Smiles 3D foi avaliado inicialmente por sete usuários, todos eles dentistas. Através de um campo de sugestões no formulário e de contato por e-mail, alguns feedbacks foram coletados, em sua maioria a relatar eventuais problemas ou a propor adições e alterações válidas na plataforma. Como por exemplo, dois usuários reportaram que seria interessante acrescentar aos pregões um campo de tipo de pagamento e um campo de tipo de entrega, outros relataram sugestões como aprimoramento visual da interface, criação de uma ferramenta de destaque, na modalidade premium, e de uma ferramenta de ranqueamento dos usuários cadastrados na aplicação, a fim de que possa ser conhecida a credibilidade e confiabilidade dos usuários registrados.

Em síntese, a plataforma foi avaliada de forma positiva, considerada principalmente acessível e útil, ainda que necessite de pequenos melhoramentos na 
utilização geral do aplicativo. A média System Usability Score teve resultado 93.2, bem acima da média de 68 pontos, o que faz concluir que a usabilidade foi bem avaliada pelos usuários. Através dos comentários fornecidos pelos dentistas, pode-se constatar que as ferramentas e alterações planejadas futuramente para o projeto são apropriadas e cruciais, tanto para o enriquecimento das funcionalidades, quanto para a facilidade de uso da plataforma como um todo.

\section{Conclusão}

O projeto Smiles 3D possui uma motivação autêntica e cada vez mais urgente em relação à sua necessidade de implementação, principalmente para os profissionais da área odontológica e para seus pacientes e clientes.

Quanto a etapa em que o projeto se apresenta, apesar de funcional, exige ainda melhorias, ainda mais específicas no que diz respeito a comunicação entre os usuários e usabilidade da plataforma. Devido ao fácil acesso à ferramenta, a Smiles 3D é considerada uma plataforma de simples visitação e registro, justamente por ser baseada em web, disponível para qualquer dispositivo cliente que possua acesso à Internet e um navegador web.

Em relação às etapas futuras, espera-se que haja a consolidação e melhor estruturação do que foi desenvolvido, aperfeiçoando as funcionalidades e as páginas web, após testes exaustivos com usuários. Será implementado em breve o cadastro de projetistas, como mais um tipo de usuário no sistema.

\section{Referências}

FERNANDES, Jorge Ulisses Jacob. (2005) Sistema de Registro de Preços Pregão Presencial e Eletrônico. 2 ed. Belo Horizonte: Fórum, p.455

PLATAFORMA BOOKLAB, http://booklab.com.br/, acessada em 10/03/2019

PLATAFORMA ODONTO COMPANY, https://www.odontocompany.com/, acessada em: 18/02/2019

PLATAFORMA DR.SINTOMAS, https://drsintomas.com.br/, acessada em: 18/02/2019

PLATAFORMA ZENKLUB, https://www.zenklub.com.br/quem-somos/, acessada em: 10/03/2019

FRAMEWORK DJANGO, Visão Global, https://www.djangoproject.com/start/, acessada em: 10/03/2019

Brooke, J. (1996). Sus: a quick and dirty usability scale. In Usability Evaluation in Industry. Taylor Francis.

\section{Vídeo de apresentação do projeto:}

https://youtu.be/7rt6xhadCJ0 